\title{
I-BET151 inhibits osteoclastogenesis via the RANKL signaling pathway in RAW264.7 macrophages
}

\author{
JING CHENG, JIFU ZHENG, NINGHONG GUO and FUMING ZI
}

Department of Hematology, The Second Affiliated Hospital of Nanchang University, Nanchang, Jiangxi 330006, P.R. China

Received November 10, 2016; Accepted July 12, 2017

DOI: $10.3892 / \mathrm{mmr} .2017 .7631$

\begin{abstract}
Excessive bone resorption mediated by osteoclasts may lead to the risk of various lytic bone diseases. In the present study, the effects of I-BET151, a bromodomain and extra terminal domain protein inhibitor, on osteoclastogenesis in RAW264.7 cells and the underlying mechanism of this process was investigated. Cells were divided into 6 groups, including the control group, receptor activator of nuclear factor- $\kappa \mathrm{B}$ ligand (RANKL) group and 4 other groups containing RANKL and I-BET151 at different concentrations. Tartrate-resistant acid phosphatase (TRACP) staining was used to observe the effect of I-BET151 on osteoclastogenesis and the number of TRACP positive multinucleated cells was calculated. Western blotting was used to evaluate the expression of tumor necrosis factor receptor associated factor (TRAF6), nuclear factor of activated T-cells cytoplasmic 1 (NFATcl), transcription factor p65 (p65), nuclear factor of $\kappa$ light polypeptide gene enhancer in B-cells inhibitor- $\alpha$ (I $\kappa \mathrm{B}-\alpha)$, extracellular signal-regulated kinase, Jun N-terminal kinase (JNK) and p38. mRNA expression levels of osteoclast specific genes TRACP, matrix metalloproteinase-9 (MMP9), cathepsin $\mathrm{K}$ (CtsK) and proto-oncogene tyrosine-protein kinase $\mathrm{Src}$ (c-Src) were measured using the reverse transcription-quantitative polymerase chain reaction (RT-qPCR). TRACP staining results demonstrated that I-BET151 inhibited osteoclastogenesis induced by RANKL and the inhibition was dose dependent. TRACP multinucleated positive cells were significantly decreased when treated with I-BET151 compared with the RANKL group. The inhibitory effect on TRAF6 was significant when concentrations of 100 and $200 \mathrm{nM}$ I-BET151 were used, and NFATcl was significantly inhibited when a concentration of $200 \mathrm{nM}$ was used compared with the RANKL group, in a dose-dependent manner. Nuclear translocation of p65 was significantly inhibited by I-BET151 at all
\end{abstract}

Correspondence to: Dr Fuming Zi, Department of Hematology, The Second Affiliated Hospital of Nanchang University, 1 Minde Road, Nanchang, Jiangxi 330006, P.R. China

E-mail: zifuming@163.com

Key words: I-BET151, osteoclastogenesis, receptor activator of nuclear factor- $\mathrm{\kappa B}$ ligand, tartrate-resistant acid phosphatase, RAW264.7 concentrations. The degradation of $\mathrm{I} \kappa \mathrm{B}-\alpha$, and phosphorylation of JNK and p38 were also significantly inhibited by I-BET151, with the exception of the expression of I $\kappa \mathrm{B}-\alpha$ following treatment with $50 \mathrm{nM}$ I-BET151. The RT-qPCR results revealed that osteoclast-specific genes TRACP, MMP9, CtsK and c-Src were all dose-dependently inhibited by I-BET151, except for CtsK. In conclusion, I-BET151 may significantly suppress the osteoclastogenesis of RAW264.7 cells via the RANKL signaling pathway.

\section{Introduction}

Bone formation and resorption are mediated by osteoblasts and osteoclasts, respectively, and regulate the balance of normal bone metabolism. Interruption of this balance may result in increased resorption compared with formation and lead to excess bone loss, causing a variety of diseases, including myeloma bone disease, osteoporosis and rheumatoid arthritis $(1,2)$. As isolating and culturing bone cells is difficult, research and molecular analysis of osteoclastogenesis has stagnated for a long period. However, developments in techniques over the past decade have allowed for further investigation $(3,4)$. There are multiple biomolecules involved in the signaling pathways of osteoclastogenesis. Among these, the most important factors are receptor activator for nuclear factor- $\kappa \mathrm{B}$ ligand (RANKL) and macrophage colony-stimulating factor (M-CSF).

Research has demonstrated that RANKL and its receptor, RANK, are necessary for osteoclastogenesis (5). These proteins activate the reconstitution of dynamic differentiation processes, including cell fusion $(5,6)$. In addition, the proteins are considered to be a possible factor involved in controlling the differentiation process. RANKL may also activate the expression of transcriptional factors such as c-Fos, microphthalmia-associated transcription factor and nuclear factor of activated T-cells cytoplasmic 1 (NFATc1), which are important for osteoclastogenesis (7). Therefore, the RANKL-RANK signaling pathway is a key signaling component involved in osteoclastogenesis.

RANK mediates signaling by recruiting adaptor molecules, including proteins of the tumor necrosis factor (TNF) receptor-associated factor (TRAF) family $(8,9)$. Studies have revealed that TRAF1 binds to RANK $(10,11)$. TRAF6 also binds to RANK, which further induces trimerization of TRAF6 and subsequently activates nuclear factor (NF)- $\mathrm{B}$ and 
mitogen-activated kinases (MAPKs) (12,13). NF- $\mathrm{B}$ is associated with the biomolecular progress of osteoclastogenesis induced by RANK and is a basic component of osteoclastogenesis activated by TRAF6 (14).

$\mathrm{M}-\mathrm{CSF}$ is another essential cytokine involved in osteoclastogenesis besides RANKL $(15,16)$. Research has demonstrated that M-CSF serves an important role in the proliferation and survival of osteoclast precursor cells (17), upregulates the expression levels of RANK, and may participate in the progression of differentiation by activating c-Fos, protein kinase $\mathrm{B}$ and extracellular signal-regulated kinase (ERK) pathways, which may interact with RANKL signals $(18,19)$.

Studies have demonstrated that bromodomain and extra terminal domain proteins (BET) serve an important role in different types of cancer, including cancer of the bones $(20,21)$. I-BET151 is a quinoline class of BET protein inhibitors, which has been demonstrated to exhibit activity against several types of cancer (22). Studies revealed that I-BET151 induced B-cell lymphoma like 11-dependent apoptosis and cell cycle arrest of human melanoma cells (23), and suppressed expression of inflammatory genes and matrix degrading enzymes in rheumatoid arthritis synovial fibroblasts (24). Research has also demonstrated that I-BET151 suppresses pathologic bone loss in TNF-induced inflammatory osteolysis (21). Despite numerous reports of I-BET151, few have focused on the role of I-BET151 in osteoclastogenesis. In the present study, the effects of I-BET151 on osteoclastogenesis and the underlying molecular signaling pathways involved were investigated.

\section{Materials and methods}

Cell culture. A mouse macrophage cell line, RAW264.7 (TIB-71 ${ }^{\mathrm{TM}}$ ), was purchased from American Type Culture Collection (Manassas, VA, USA). RAW264.7 cells were cultured in Dulbecco's modified Eagle's medium (Thermo Fisher Scientific, Inc., Waltham, MA, USA) supplemented with $10 \%$ fetal bovine serum (Gibco; Thermo Fisher Scientific, Inc.) and $100 \mu \mathrm{g} / \mathrm{ml}$ penicillin-streptomycin (Sigma-Aldrich; Merck KGaA, Darmstadt, Germany). RANKL (PeproTech, Inc., Rocky Hill, NJ, USA) was diluted to $100 \mathrm{ng} / \mathrm{ml}$ in aquae sterilisata. I-BET151 (MedChemExpress, Monmouth Junction, NJ, USA) was dissolved in dimethyl sulfoxide at a concentration of $<0.1 \%$ (Sigma-Aldrich; Merck KGaA). RAW264.7 cells were seeded in 6-well plates $\left(2 \times 10^{7}\right.$ cells/well) in a humidified incubator at $5 \% \mathrm{CO}_{2}$ and $37^{\circ} \mathrm{C}$, supplemented with $100 \mathrm{ng} / \mathrm{ml}$ RANKL, and treated with different concentrations of I-BET151 (25). Cells were cultured for 7 days to induce osteoclast differentiation. The study was divided into 6 groups: Control; RANKL (100 ng/ml); RANKL (100 ng/ml) and I-BET151 (50 nM); RANKL (100 ng/ml) and I-BET151 (100 nM); RANKL (100 ng/ml) and I-BET151 (200 nM); and RANKL (100 ng/ml) and I-BET151 (400 nM).

TRACP staining. TRACP staining was used to determine the effect of I-BET151 on osteoclastogenesis and the percentage of TRACP positive multinucleated cells was calculated. Cells were fixed using freshly made, refrigerated, 3\% paraformaldehyde (PFA; Sigma-Aldrich; Merck KGaA) and 2\% sucrose in phosphate-buffered saline (PBS; Sigma-Aldrich, Merck KGaA) for $10 \mathrm{~min}$ and stained for TRACP using a tartrate-resistant acid phosphatase stain kit according to manufacturer's protocol (Nanjing Jiangcheng Bioengineering Institute, Nanjing, China), after 7 days culture in the presence of RANKL and different concentrations of I-BET151. TRACP positive multinucleated cells ( $>3$ nuclei) were counted under 8 fields of view for each sample and regarded as osteoclasts (26). Cells were observed using a Zeiss Axio Observer D1 microscope with $\times 100$ magnification and images were captured and analyzed with Zeiss ZEN software version 2012 (Zeiss GmbH, Jena, Germany).

Reverse transcription-quantitative polymerase chain reaction $(R T-q P C R)$. RAW264.7 cells $\left(2 \times 10^{4}\right)$ were incubated with RANKL $(100 \mathrm{ng} / \mathrm{ml})$ and different concentrations of I-BET151 for 4 days. RT-qPCR was employed to determine the expression levels of osteoclastic-specific marker genes and GAPDH was used as a control. RNA extraction and reverse-transcription were performed as described previously (27). Total RNA was extracted using TRIzol reagent (Invitrogen; Thermo Fisher Scientific, Inc.) according to the manufacturer's protocol. A High Capacity cDNA Reverse Transcription kit (Applied Biosystems; Thermo Fisher Scientific, Inc.) was used to convert RNA into cDNA. qPCR was conducted using a ABI 7500 real-time PCR instrument (Applied Biosystems; Thermo Fisher Scientific, Inc.) with SYBR Premix Ex Taq (Takara Biotechnology Co., Ltd., Dalian, China). Primers used in PCR were as follows: Primers for TRACP were: Forward, 5'-ACA CAGTGATGCTGTGTGGCAACTC-3' and reverse, 5'-CCA GAGGCTTCCACATATATGATGG-3'; Primers for MMP9 were: Forward, 5'-AGTTTGGTGTCGCGGAGCAC-3' and reverse, 5'-TACATGAGCGCTTCCGGCAC-3'; Primers for CtsK were: Forward, 5'-GGCCAACTCAAGAAGAAAAC-3' and reverse, 5'-GTGCTTGCTTCCCTTCTGG-3; Primers for c-Src were: Forward, 5'-CCAGGCTGAGGAGTGGTACT-3' and reverse, 5'-CAGCTTGCGGATCTTGTAGT-3'; Primers for GAPDH were: Forward, 5'-AACTTTGGCATTGTGGAA GG-3' and reverse, 5'-ACACATTGGGGGTAGGAACA-3'. DNA was denatured at $94^{\circ} \mathrm{C}$ for $10 \mathrm{~min}$, followed by initial denaturation with 30 cycles at $94^{\circ} \mathrm{C}$ for $1 \mathrm{~min}, 60^{\circ} \mathrm{C}$ for $1 \mathrm{~min}$ and $72^{\circ} \mathrm{C}$ for $2 \mathrm{~min}$, and finally ended up with an extension step at $72^{\circ} \mathrm{C}$ for $5 \mathrm{~min}$. Relative quantification of RT-qPCR product was performed using the comparative $2^{-\Delta \Delta \mathrm{Cq}}$ method (28).

Western blotting. Western blotting was used to determine the expression levels of TRAF6, NFATc 1, and the influence of I-BET151 on the NF- $\kappa$ B signaling pathway (p65 and I $\kappa$ B- $\alpha$ ) and MAPK signaling pathway [ERK, Jun N-terminal kinase (JNK) and p38]. A Nuclear Extraction kit was purchased from Cayman Chemical Company (Ann Arbor, MI, USA) to use for measurement of p65. $\beta$-actin was used as a loading control. Samples extracted from the cells as previously described (29) were loaded on $10 \%$ SDS-PAGE, prior to transfer onto polyvinylidene difluoride membranes. Membranes were blocked with 5\% non-fat milk in TBS containing $0.1 \%$ Tween-20 for $2 \mathrm{~h}$ at room temperature. Subsequently, membranes were probed with primary antibodies at $4^{\circ} \mathrm{C}$ overnight and a horseradish peroxidase (HRP) conjugated secondary antibody for $2 \mathrm{~h}$ at room temperature (Cell Signaling Technology, Inc., Danvers, MA, USA). The membranes were incubated with an Enhanced Chemiluminescence detection 

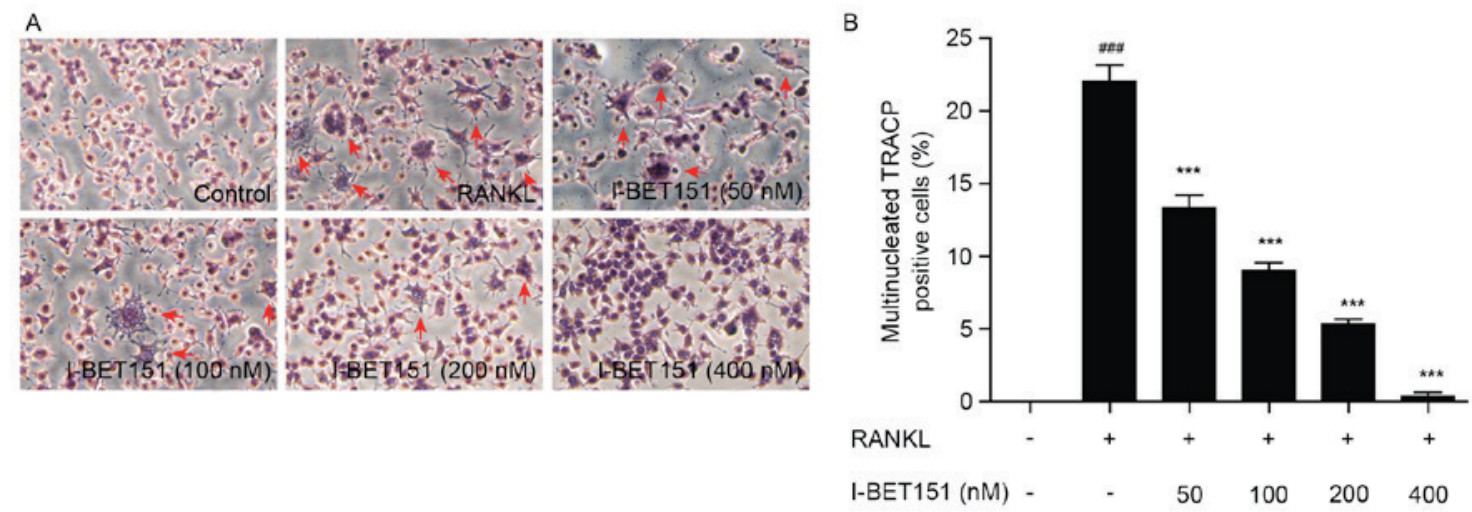

Figure 1. (A) TRACP staining of RAW264.7 cells. (B) Quantification of multinucleated TRACP positive cells. Representative images from each group are demonstrated. Arrows indicate TRACP positive multinucleated cells. ${ }^{\# \#} \mathrm{P}<0.001$ vs. control group; ${ }^{* * *} \mathrm{P}<0.001$ vs. RANKL group. TRACP, tartrate-resistant acid phosphatase; RANKL, receptor activator of nuclear factor- $\mathrm{\kappa}$ ligand.

kit (Amersham Pharmacia Biotech AB, Uppsala, Sweden) and were exposed to X-ray film. The films were scanned and proteins were quantified using Quantity One software version 4.2.1 (Bio-Rad Laboratories, Inc., Hercules, CA, USA). Antibodies used in western blotting were as follows: Anti-TRAF6 (ab33915; 1:1,000; Abcam, Cambridge, UK); NFATC1 antibody (MA3-024; 1:2,000; Thermo Fisher

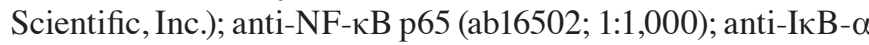
(E130; ab32518; 1:1,000); anti-ERK1+ERK2 (ab17942; 1:1,000); anti-ERK 1/2 (phospho-Thr202/Tyr204; ab214362; 1:1,000); anti-JNK1+JNK2+JNK3 (ab179461; 1:1,000); anti-JNK1+JNK2+JNK3 (phospho T183+T183+T221; ab124956; 1:1,000); anti-p38 (ab31828; 1:1,000); anti-p38 (phospho Y182; ab47363; 1:1,000); anti- $\beta$-actin (ab8226; $1: 1,000)$; and anti-mouse $\operatorname{IgG}$ VeriBlot for IP secondary (HRP-conjugated; ab131368; 1:2,000) (all from Abcam).

Statistical analysis. Data are expressed as the mean \pm standard deviation. Independent continuous variables were compared using a Student's t-test for comparison of two groups or a one-way analysis of variance followed by Tukey post hoc test for multiple comparison. $\mathrm{P}<0.05$ was considered to indicate a statistically significant difference. All calculations were made using SPSS software version 18.0 (SPSS, Inc., Chicago, IL, USA).

\section{Results}

TRACP staining. TRACP staining was used to determine the effect of I-BET151 on osteoclastogenesis and the percentage of TRACP multinucleated positive cells was calculated. Results revealed that in the RANKL group, multiple cell fusion increased the cell volumes and cell synapse, and resulted in multinucleated cells, which was inhibited by I-BET151 (Fig. 1). In the control group, osteoclastogenesis proceeded normally; however, this was enhanced in the RANKL group. When treated with I-BET151, it was apparent that osteoclastogenesis induced by RANKL was dose-dependently inhibited (Fig. 1). Fig. 1B demonstrates the percentage TRACP positive cells. Compared with the control group, the percentage of TRACP positive cells in groups 2-5 was increased (Fig. 1). However, the percentage of TRACP positive cells was almost completely abolished following treatment with $400 \mathrm{nM}$ I-BET151. The percentage of TRACP positive cells in all groups treated with I-BET151 was significantly decreased compared with the RANKL group $(\mathrm{P}<0.001$; Fig. 1$)$ and the inhibitory effect was dose dependent.

Effect of I-BETI5I on the RANKL signaling pathway. The expression levels of TRAF6 and NFATc 1, which are upstream and downstream effectors of the RANKL signaling pathway, respectively, were measured by western blotting (Fig. 2). TRAF6 and NFATc 1 were dose-dependently inhibited by I-BET151. Densitometric analysis of the TRAF6/ $\beta$-actin ratio in the I-BET151 100 and $200 \mathrm{nM}$ groups were significantly decreased compared with the RANKL group, whereas the NFATc $1 / \beta$-actin ratio was only significantly decreased compared with the RANKL group following treatment with $200 \mathrm{nM}$ I-BET151. TRAF6/ $\beta$-actin and NFATc $1 / \beta$-actin in the $50 \mathrm{nM}$ I-BET151 group demonstrated no significant difference compared with the RANKL group. The NFATc $1 / \beta$-actin ratio and TRAF6/ $\beta$-actin ratio in the RANKL group was significant increased compared with the control group. The inhibition effect of both TRAF6/ $\beta$-actin and NFATc $1 / \beta$-actin by I-BET151 were dose-dependent.

Effect of I-BET151 on the NF- $\kappa B$ and MAPK signaling pathways. The expression levels of $\mathrm{p} 65$ and IкB- $\alpha$ (involved in the NF- $\mathrm{BB}$ signaling pathway) and ERK, JNK and p38 (involved in the MAPK signaling pathway) were evaluated by western blotting. Results revealed that nuclear expression of p65 was significantly inhibited by I-BET151 (Fig. 3A and B) at concentrations of 50, 100 and $200 \mathrm{nM}$ compared with the RANKL group, and the effect increased as the dose increased. In addition, under concentrations of 100 and 200 nM I-BET151, IкB- $\alpha$ was significantly inhibited compared with cells treated with RANKL alone (Fig. 3A and C). However, expression of IкB- $\alpha$ after treatment with $50 \mathrm{nM}$ I-BET151 did not demonstrate a significant difference compared with the RANKL group (Fig. 3C). Phosphorylation of JNK and p38 was significantly inhibited by 50,100 and $200 \mathrm{nM}$ I-BET151 compared with the RANKL group (Fig. 3D, E and F). By contrast, only $50 \mathrm{nM}$ I-BET151 markedly inhibited the expression levels 
A

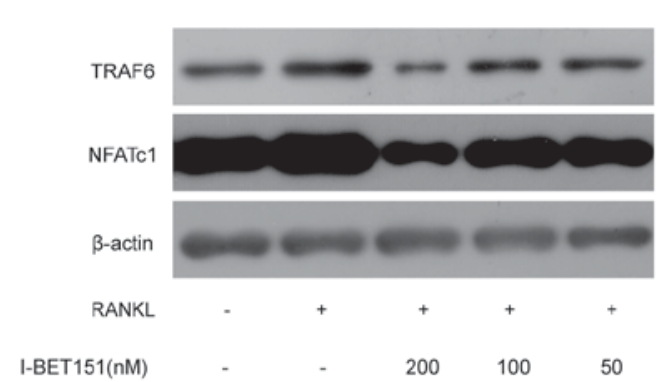

B

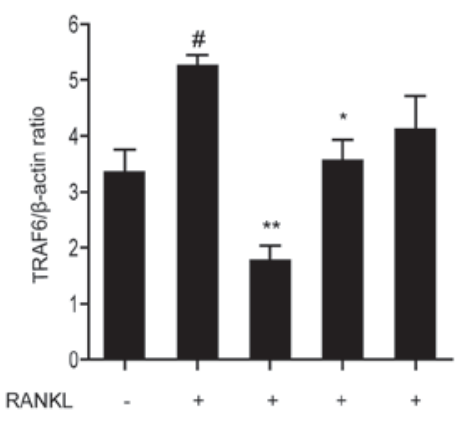

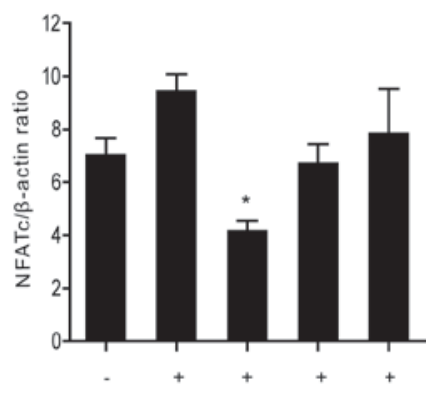

$200 \quad 100 \quad 50$

Figure 2. (A) Western blotting of TRAF6 and NFATc1. (B) Quantified expression levels of TRAF6 and NFATc1 protein in RAW264.7 cells treated with or without RANKL, and with or without I-BET151 at 200,100 or $50 \mathrm{nM}$. ${ }^{*} \mathrm{P}<0.05$ vs. control group; ${ }^{*} \mathrm{P}<0.05,{ }^{* *} \mathrm{P}<0.01$ vs. RANKL group. TRAF6, tumor necrosis factor receptor-associated factor 6; RANKL, receptor activator of nuclear factor-кB ligand; NFATc1, nuclear factor of activated T-cells cytoplasmic 1.

A

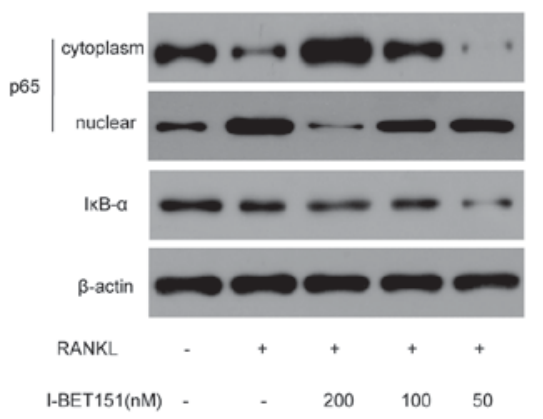

B

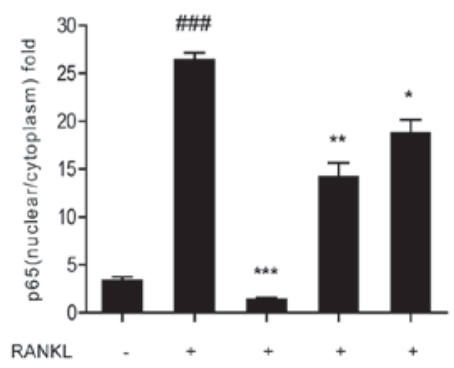

I-BET151(nM) -
C

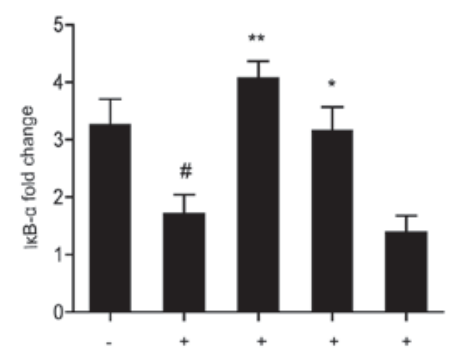

D

E

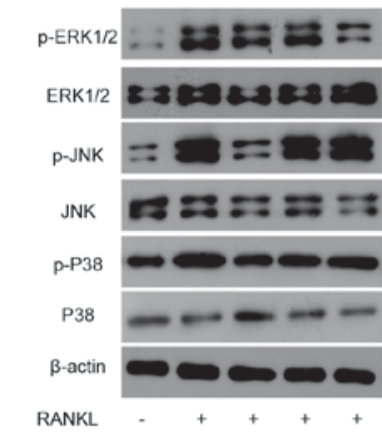

$\begin{array}{lllll}\text { RANKL } & + & + & + & + \\ \text { I-BET151(nM) - } & - & 200 & 100 & 50\end{array}$

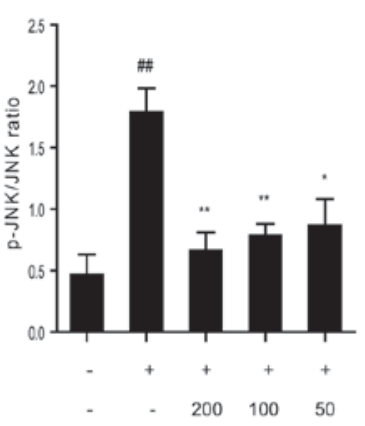

F

G
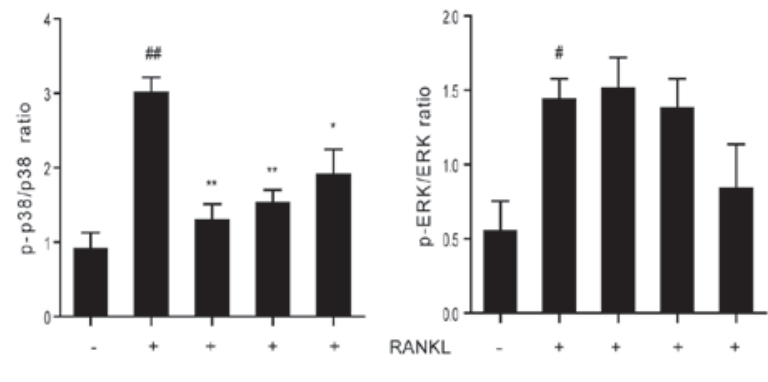

20010050 I-BET151(nM) -

$200 \quad 100 \quad 50$

Figure 3. (A) Western blotting of p65 and IкB- $\alpha$. RAW264.7 cells treated with or without RANKL. and with or without I-BET151 at 200,100 or $50 \mathrm{nM}$. Densitometric analysis of (B) nuclear/cytoplasmic p65 and (C) IкB- $\alpha$. (D) Western blotting of total or phosphorylated ERK, JNK and p38. Densitometric analysis of (E) p-JNK/JNK, (F) p-p38/p38 and (G) p-ERK/ERK. ${ }^{\#} \mathrm{P}<0.05,{ }^{* \#} \mathrm{P}<0.01,{ }^{\# \# "} \mathrm{P}<0.001$ vs. control group; ${ }^{*} \mathrm{P}<0.05,{ }^{* * *} \mathrm{P}<0.01,{ }^{* * *} \mathrm{P}<0.01$ vs. RANKL group. RANKL, receptor activator of nuclear factor- $\kappa \mathrm{B}$ ligand; IкB- $\alpha$, nuclear factor of $\kappa$ light polypeptide gene enhancer in B-cells inhibitor- $\alpha$; ERK, extracellular signal-regulated kinase; JNK, Jun N-terminal kinase; p-JNK, phosphorylated Jun N-terminal kinase; p-p38, phosphorylated p38; p-ERK, phosphorylated extracellular signal-regulated kinase. 
A

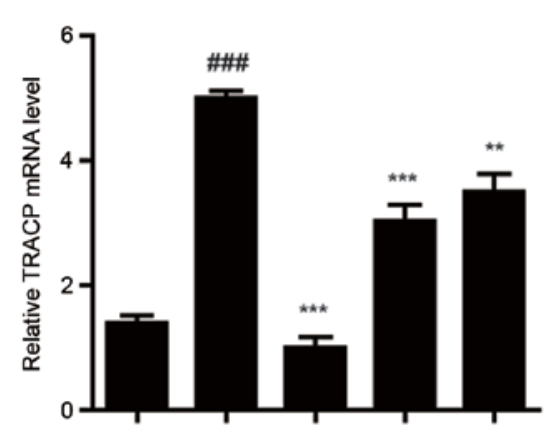

C

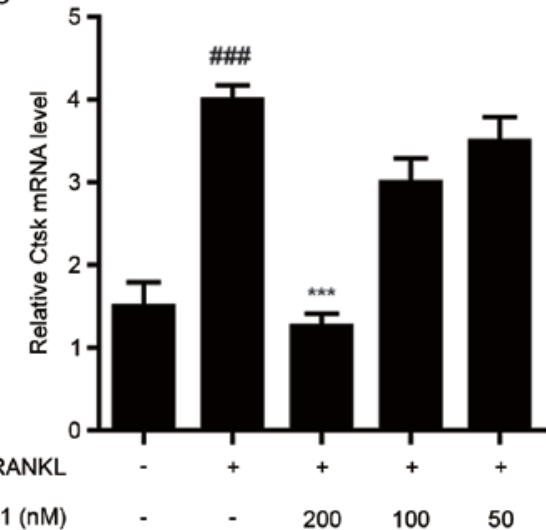

B
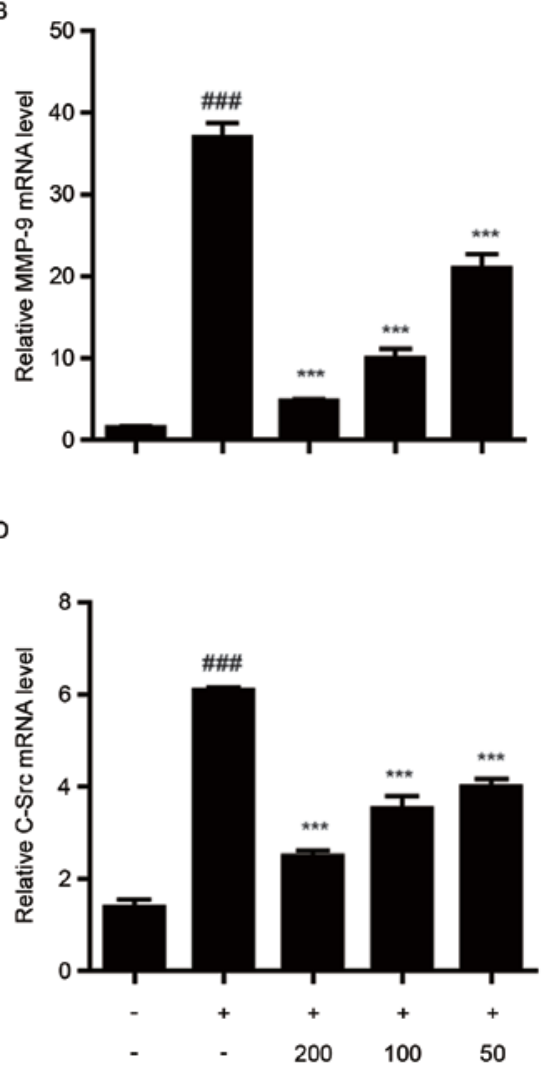

Figure 4. mRNA expression levels of the osteoclast-specific genes (A) TRACP, (B) MMP9, (C) CtsK and (D) c-Src using the reverse transcription-quantitative polymerase chain reaction. RAW264.7 cells treated with or without RANKL and with or without I-BET151 at 200,100 or 50 nM. ${ }^{\# \#} \mathrm{P}<0.001$ vs. control group; ${ }^{* * *} \mathrm{P}<0.01,{ }^{* * *} \mathrm{P}<0.001$ vs. RANKL group. TRACP, tartrate-resistant acid phosphatase; RANKL, receptor activator of nuclear factor- $\kappa \mathrm{B}$ ligand; MMP9, matrix metallopeptidase 9; CtsK, cathepsin K; c-Src, proto-oncogene tyrosine-protein kinase Src.

of p-ERK compared with the RANKL group; however, no significant difference was observed following treatment with I-BET151 (Fig. 3D and G).

Effect of I-BET151 on the expression of osteoclast-specific genes. The mRNA expression levels of the genes encoding osteoclast-specific proteins TRACP, matrix metalloproteinase-9 (MMP9), cathepsin K (CtsK) and c-Src were measured using RT-qPCR. The results revealed that all genes were dose-dependently inhibited by I-BET151 compared with the RANKL group. With the exception of CtsK, the inhibitory effects on TRACP, MMP9, and c-Src at all concentrations of I-BET151 were statistically significant compared with the RANKL group (Fig. 4). For Ctsk, only 200 nM I-BET151 led to significant inhibition in expression compared with the RANKL group.

\section{Discussion}

A delicate balance exists in the process of bone remodeling between bone formation and resorption (30). Excessive bone resorption may lead to over activation of osteoclasts and be a risk of various lytic bone diseases, including rheumatoid arthritis, psoriatic arthritis and osteoporosis (31). RANKL serves a role in the process of osteoclastogenesis. In the RANKL signaling pathway, a number of biomolecules are involved and have their own role, including TRAF6, NF- $\kappa$ B and MAPKs. NF- $\kappa \mathrm{B}$ is essential for the initial induction or autoamplification of NFATc1, which may activate other osteoclast-specific genes including TRACP, CtsK, calcitonin receptor and MMP9. Although there are various studies focusing on I-BET151, few report the effects of BET on the inhibition of osteoclastogenesis. Park-Min et al (21) reported that I-BET151 suppresses pathologic bone loss in TNF-induced inflammatory osteolysis and suppresses osteoclastogenesis; however, the detailed effects of I-BET151 on the RANKL signaling pathway, and its associated proteins and genes involved in this process, remain to be identified. In the present study, the effects of I-BET151 on osteoclastogenesis were investigated by determining the expression levels of TRAF6 and NFATcl that are involved in RANKL pathway, and the effects of I-BET151 on osteoclast-specific gene expression.

According to the TRACP staining results, I-BET151 inhibited RANKL-induced osteoclastogenesis and the inhibition was demonstrated to be dose dependent. Also, the percentage of TRACP multinucleated-positive cells was reduced when treated with I-BET151, and the effect was significant compared with the RANKL group. Expression of TRAF6 and NFATc, which are upstream and downstream effectors of the RANKL pathway were dose-dependently inhibited by I-BET151, which was consistent with the research by Park-Min et al (21). In the present study, at concentrations of 100 and $200 \mathrm{nM}$ I-BET151, the inhibitory effect of TRAF6 was significant, as was the inhibitory effect for NFATcl at 
200 nM I-BET151. Studies have demonstrated that there are several inhibitors of the RANKL signaling pathway. Meng et al (25) reported that another BET inhibitor, JQ1, may significantly suppress RANKL-induced osteoclast markers, such as c-Fos, NFATcl, TRAP and CtsK, as well as toll-like receptor (TLR)2/4, NF- $\kappa \mathrm{B}$ phosphorylation and nuclear translocation. Li et al (29) studied the effect of sinomenine on TLR4/TRAF6 expression, and the results revealed that sinomenine reduces the expression levels of RANK adaptor molecule TRAF6 and downregulates RANKL-induced $\mathrm{NF}-\kappa \mathrm{B}$ activation.

Further effects of I-BET151 on expression of p65 and I $\kappa$ B $-\alpha$ (involved in NF- $\kappa$ B pathway) and ERK, JNK and p38 (involved in MAPK pathway) were also evaluated in the present study. Results revealed that nuclear expression of p65 was significantly inhibited by I-BET151 at all concentrations. Degradation of I $\mathrm{B}-\alpha$ and phosphorylation of JNK and p38 was also significantly inhibited by I-BET151, with the exception of I $\kappa \mathrm{B}-\alpha$ expression after treatment with I-BET151 at $50 \mathrm{nM}$, which did not exhibit a significant difference compared with the RANKL group. These results were also consistent with the effects of JQ1 (25) and sinomenine (27,29). However, I-BET151 did not exhibit a significant inhibitory effect on ERK, and this result was consistent with the effect of sinomenine (29).

Klein et al (24) reported that I-BET151 may suppress cytokine and TLR ligand-induced secretion of MMP1, MMP3, interleukin (IL)-6 and IL-8, and mRNA expression of $\geq 70 \%$ of the genes induced by TNF- $\alpha$ and IL-1 $\beta$. In the present study, the mRNA expression levels of the genes encoding osteoclast-specific proteins TRACP, MMP9, CtsK and c-Src were measured. The results demonstrated that all genes were dose-dependently inhibited by I-BET151. With the exception of CtsK, the inhibitory effects of TRACP, MMP9 and c-Src were significant compared with the RANKL group.

In conclusion, the influence of I-BET151 on osteoclastogenesis in RAW264.7 cells was investigated in the present study. The results revealed that the BET inhibitor, I-BET151, significantly suppressed osteoclastogenesis of RAW264.7 cells, possibly via the RANKL signaling pathway. Based on the present literature, to the best of the authors' knowledge, there is no previous study focusing on the effect of I-BET151 on the osteoclastogenesis of RAW264.7 cells and the possible associated pathways, including RANKL, NF- $\kappa$ B and MAPK.

\section{Acknowledgements}

The work of the present study was supported by the Science and Technology Planning Project of Health and Family Planning Commission of Jiangxi Province in 2016 (grant nos. 20161066 and 20161067).

\section{References}

1. Weivoda MM, Ruan M, Hachfeld CM, Pederson L, Howe A, Davey RA, Zajac JD, Kobayashi Y, Williams BO, Westendorf JJ, et al: Wnt signaling inhibits osteoclast differentiation by activating canonical and noncanonical cAMP/PKA pathways. J Bone Miner Res 31: 65-75, 2016.

2. Longo V, Brunetti O, D'Oronzo S, Dammacco F and Silvestris F: Therapeutic approaches to myeloma bone disease: An evolving story. Cancer Treat Rev 38: 787-797, 2012.
3. Delaisse JM B A, Ali NN, et al: The effects of both cysteine proteinase and collagenase inhibitors on dentine resorption by isolated osteoclasts-Bone. Bone 44: 45-46, 2014.

4. Bara JJ, Richards RG, Alini M and Stoddart MJ: Concise review: Bone marrow-derived mesenchymal stem cells change phenotype following in vitro culture: Implications for basic research and the clinic. Stem Cells 32: 1713-1723, 2014.

5. Klein-Nulend J, Bakker AD, Bacabac RG, Vatsa A and Weinbaum S: Mechanosensation and transduction in osteocytes. Bone 54: 182-190, 2013.

6. Zhai ZJ, Li HW, Liu GW, Qu XH, Tian B, Yan W, Lin Z, Tang TT, Qin A and Dai KR: Andrographolide suppresses RANKL-induced osteoclastogenesis in vitro and prevents inflammatory bone loss in vivo. Br J Pharmacol 171: 663-675, 2014.

7. Cicek M, Vrabel A, Sturchio C, Pederson L, Hawse JR, Subramaniam M, Spelsberg TC and Oursler MJ: TGF- $\beta$ inducible early gene 1 regulates osteoclast differentiation and survival by mediating the NFATc1, AKT and MEK/ERK signaling pathways. Plos One 6: e17522, 2011.

8. D'Amelio P, Isaia G and Isaia GC: The osteoprotegerin/RANK/RANKL system: A bone key to vascular disease. J Endocrinol Invest 32 (4 Suppl): S6-S9, 2014.

9. Jules J, Wang SQ, Shi ZQ, Liu JZ, Wei S and Feng X: The IVVY motif and tumor necrosis factor receptor-associated factor (TRAF) sites in the cytoplasmic domain of the receptor activator of nuclear factor $\kappa \mathrm{B}$ (RANK) cooperate to induce osteoclastogenesis. J Biol Chem 290: 23738-23750, 2015.

10. Galibert L, Tometsko ME, Anderson DM, Cosman D and Dougall WC: The involvement of multiple tumor necrosis factor receptor (TNFR)-associated factors in the signaling mechanisms of receptor activator of NF-kappaB, a member of the TNFR superfamily. J Biol Chem 273: 34120-34127, 1999.

11. Wong BR, Josien R, Lee SY, Vologodskaia M, Steinman RM and Choi Y: The TRAF family of signal transducers mediates NF-kappaB activation by the trance receptor. J Biol Chem 273: 28355-28359, 1998.

12. Liu H, Tamashiro S, Baritaki S, Penichet M, Yu Y, Chen H, Berenson $\mathrm{J}$ and Bonavida B: TRAF6 activation in multiple myeloma: A potential therapeutic target. Clin Lymphoma Myeloma Leuk 12: 155-163, 2012.

13. Kobayashi N, Kadono Y, Naito A, Matsumoto K, Yamamoto T, Tanaka S and Inoue J: Segregation of TRAF6-mediated signaling pathways clarifies its role in osteoclastogenesis. Embo Journal 20: 1271-1280, 2001.

14. Guan H, Mi B, Li Y, Wu W, Tan P, Fang Z, Li J, Zhang Y and Li F: Decitabine represses osteoclastogenesis through inhibition of RANK and NF-kB. Cell Signal 27: 969-977, 2015.

15. De Vries TJ, Schoenmaker T, Aerts D, Grevers LC, Souza PP, Nazmi K, van de Wiel M, Ylstra B, Lent PL, Leenen PJ and Everts V: M-CSF priming of osteoclast precursors can cause osteoclastogenesis-insensitivity, which can be prevented and overcome on bone. J Cell Physiol 230: 210-225, 2015.

16. Wikto-rjedrzejczak W, Bartocci A, Ferrante AW Jr, Ahmed-Ansari A, Sell KW, Pollard JW and Stanley ER: Total absence of colony-stimulating factor 1 in the macrophage-deficient osteopetrotic (op/op) mouse. Proc Natl Acad Sci USA 87: 4828-4832, 1990.

17. Kim HJ, Yoon HJ, Yoon KA, Gwon MR, Jin Seong S, Suk K, Kim SY and Yoon YR: Lipocalin-2 inhibits osteoclast formation by suppressing the proliferation and differentiation of osteoclast lineage cells. Exp Cell Res 334: 301-309, 2015.

18. Goettsch C, Babelova A, Trummer O, Erben RG, Rauner M, Rammelt S, Weissmann N, Weinberger V, Benkhoff S, Kampschulte M, et al: NADPH oxidase 4 limits bone mass by promoting osteoclastogenesis. J Clin Invest 123: 4731-4738, 2013.

19. Ross FP and Teitelbaum SL: Alphavbeta 3 and macrophage colony-stimulating factor: Partners in osteoclast biology. Immunol Rev 208: 88-105, 2005.

20. Lamoureux F, Baud'huin M, Calleja LR, Jacques C, Berreur M, Rédini F, Lecanda F, Bradner JE, Heymann D and Ory B: Selective inhibition of BET bromodomain epigenetic signalling interferes with the bone-associated tumour vicious cycle. Nature Communications 5, 2014.

21. Park-Min KH, Lim E, Lee MJ, Park SH, Giannopoulou E, Yarilina A, van der Meulen M, Zhao B, Smithers N, Witherington J, et al: Inhibition of osteoclastogenesis and inflammatory bone resorption by targeting BET proteins and epigenetic regulation. Nat Commun 5: 5418, 2014. 
22. Chaidos A, Caputo V, Gouvedenou K, Liu B, Marigo I, Chaudhry MS, Rotolo A, Tough DF, Smithers NN, Bassil AK, et al: Potent antimyeloma activity of the novel bromodomain inhibitors I-BET151 and I-BET762. Blood 123: 697-705, 2014.

23. Gallagher SJ, Mijatov B, Gunatilake D, Tiffen JC, Gowrishankar K, Jin L, Pupo GM, Cullinane C, Prinjha RK, Smithers N, et al: The epigenetic regulator I-BET151 induces BIM-dependent apoptosis and cell cycle arrest of human melanoma cells. J Invest Dermatol 134: 2795-2805, 2014.

24. Klein K, Kabala PA, Grabiec AM, Gay RE, Kolling C, Lin LL, Gay S, Tak PP, Prinjha RK, Ospelt C and Reedquist KA: The bromodomain protein inhibitor I-BET151 suppresses expression of inflammatory genes and matrix degrading enzymes in rheumatoid arthritis synovial fibroblasts. Ann Rheum Dis 75 422-429, 2016.

25. Meng S, Zhang L, Tang Y, Tu Q, Zheng L, Yu L, Murray D, Cheng J, Kim SH, Zhou X and Chen J: BET inhibitor JQ1 blocks inflammation and bone destruction. J Dent Res 93: 657-662, 2014.

26. Wu X, Li Z, Yang Z, Zheng C, JingJ, Chen Y, Ye X, Lian X, Qiu W, Yang F, et al: Caffeic acid 3,4-dihydroxy-phenethyl ester suppresses receptor activator of NF- $\mathrm{\kappa B}$ ligand-induced osteoclastogenesis and prevents ovariectomy-induced bone loss through inhibition of mitogen-activated protein kinase/activator protein 1 and $\mathrm{Ca}^{2+}$-nuclear factfactor of activated T-cells cytoplasmic 1 signaling pathways. J Bone Miner Res 27: 1298-1308, 2012.
27. He LG, Duan H, Li XL, Wang S, Zhang Y, Lei L, Xu J, Liu S and Li X: Sinomenine down-regulates TLR4/TRAF6 expression and attenuates lipopolysaccharide-induced osteoclastogenesis and osteolysis. Eur J Pharmacol 779: 66-79, 2016.

28. Livak KJ and Schmittgen TD: Analysis of relative gene expression data using real-time quantitative PCR and the 2(-Delta Delta C(T)) method. Methods 25: 402-408, 2001.

29. Li X, He L, Hu Y, Duan H, Li X, Tan S, Zou M, Gu C, Zeng X, Yu L, et al: Sinomenine suppresses osteoclast formation and mycobacterium tuberculosis H37Ra-induced bone loss by modulating RANKL signaling pathways. Plos One 8 , 2013.

30. Kular J, Tickner J, Chim SM and Xu JK: An overview of the regulation of bone remodelling at the cellular level. Clin Biochem 45: 863-873, 2012

31. Deal C: Bone loss in rheumatoid arthritis: Systemic, periarticular and focal. Curr Rheumatol Rep 14: 231-237, 2012. 